\title{
Editorial Note: Emerging Immersive Multimedia Techniques
}

Multimedia Tools and Applications gratefully acknowledges the editorial work of the scholars listed below on the special issue entitled, "Emerging Immersive Multimedia Techniques."

\section{Prof. James Park (Corresponding Guest Editor)}

Seoul National University of Science and Technology, Korea

E-mail: jamespark.seoul@gmail.com

Website: http://www.parkjonghyuk.net

\section{Prof. Yi Pan}

Georgia State University, USA

E-mail: yipan@gsu.edu

Website: http://www.cs.gsu.edu/pan

\section{Prof. Jianhua Ma}

Hosei University

E-mail: jianhua@hosei.ac.jp

Website: http://cis.k.hosei.ac.jp/ jianhua/ 